JOURNAL OF SYNCHROTRON RADIATION

ISSN 1600-5775

Received 1 October 2017

Accepted 21 December 2017

Edited by S. Svensson, Uppsala University, Sweden

Keywords: time-stamping camera; velocity map imaging; VMI; free-electron laser; FEL; ultrafast laser; pump-probe experiments; mass spectrometry.

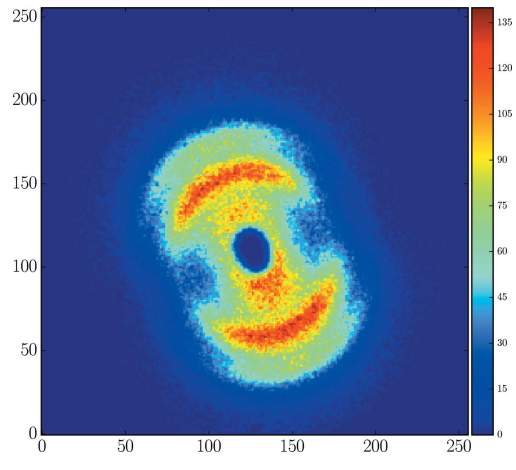

C 2018 International Union of Crystallography

\section{Time-resolved ion imaging at free-electron lasers using TimepixCam}

\author{
Merlin Fisher-Levine, ${ }^{\mathrm{a}, \mathrm{b}}$ Rebecca Boll, ${ }^{\mathrm{c}, \mathrm{d}}$ Farzaneh Ziaee, ${ }^{\mathrm{e}}$ Cédric Bomme, ${ }^{\mathrm{c}}$ \\ Benjamin Erk, ${ }^{\mathrm{c}}$ Dimitrios Rompotis, ${ }^{\mathrm{c}}$ Tatiana Marchenko, ${ }^{\mathrm{f}}$ Andrei Nomerotski ${ }^{\mathrm{a} *}$ \\ and Daniel Rolles ${ }^{\mathrm{e}} *$
}

\footnotetext{
a Brookhaven National Laboratory, Upton, NY 11973, USA, 'b Princeton University, Princeton, NJ 08544, USA, ${ }^{\mathbf{c}}$ Deutsches Elektronen-Synchrotron (DESY), 22607 Hamburg, Germany, ${ }^{\mathbf{d} E u r o p e a n ~ X F E L ~ G m b H, ~} 22869$ Schenefeld, Germany, ' J. R. Macdonald Laboratory, Department of Physics, Kansas State University, Manhattan, KS 66506, USA, and 'Sorbonne Universités, UPMC Université Paris 06, CNRS, UMR 7614, Laboratoire de Chimie Physique-Matière et Rayonnement, F-75005 Paris, France. *Correspondence e-mail: anomerotski@bnl.gov, rolles@phys.ksu.edu
}

The application of a novel fast optical-imaging camera, TimepixCam, to molecular photoionization experiments using the velocity-map imaging technique at a free-electron laser is described. TimepixCam is a $256 \times 256$ pixel CMOS camera that is able to detect and time-stamp ion hits with $20 \mathrm{~ns}$ timing resolution, thus making it possible to record ion momentum images for all fragment ions simultaneously and avoiding the need to gate the detector on a single fragment. This allows the recording of significantly more data within a given amount of beam time and is particularly useful for pump-probe experiments, where drifts, for example, in the timing and pulse energy of the free-electron laser, severely limit the comparability of pump-probe scans for different fragments taken consecutively. In principle, this also allows ion-ion covariance or coincidence techniques to be applied to determine angular correlations between fragments.

\section{Introduction}

The increased availability of short-pulse extreme ultraviolet (XUV) and (soft) X-ray sources such as free-electron lasers (FELs) (Ackermann et al., 2007; Shintake et al., 2008; Feldhaus, 2010; Emma et al., 2010; Allaria et al., 2012, 2013; Ishikawa et al., 2012; Ullrich et al., 2012) and high-order harmonic generation (HHG) sources (Popmintchev et al., 2010; Chini et al., 2014; Hädrich et al., 2014), accompanied by continuing advances in femtosecond laser technology, promises a new era for femtosecond pump-probe experiments studying the dynamics of photochemical reactions in gas-phase molecules. Many of these experiments are performed using the velocitymap imaging (VMI) technique (Eppink \& Parker, 1997), where the photoelectrons or fragment ions that are created by the ionizing light pulse are accelerated by an electric field and projected onto phosphor screen detectors that are commonly read out by CCD or CMOS cameras. Since these cameras are not fast enough to resolve the time-of-flight differences between different ion species, which typically are in the range of hundreds of nanoseconds to a few microseconds, most experiments are performed by rapidly applying a high-voltage gate to the ion detector for a limited time in order to record images for an ion with a specific mass-over-charge ratio. This means that only one ion species can be recorded at a time, and the pump-probe scans therefore have to be repeated multiple times if more than one species is to be investigated. This significantly increases the required duration of experiments, 
and hence imposes strict requirements on their stability so that different ion species can be compared.

An alternative detection technique that allows simultaneous recording and resolving of different ion species is based on multi-hit delay-line detectors, which are commonly used in so-called COLTRIMS or 'reaction microscope' setups (Dörner et al., 2000; Ullrich et al., 2003) but can also be used for VMI spectrometers (Rolles et al., 2007; Pesic et al., 2007; Ablikim et al., 2016). However, delay-line detectors can only resolve a small number of particle hits within a short time-offlight window (a few hits per $100 \mathrm{~ns}$ at best), which significantly limits the count rate and poses a serious problem for experiments with low-repetition rate and/or high-intensity sources.

Here we describe the application of a novel fast opticalimaging camera, TimepixCam (Fisher-Levine \& Nomerotski, 2016), in conjunction with a VMI spectrometer for a timeresolved ion-imaging experiment performed at the free-electron laser FLASH at DESY in Hamburg (Ackermann et al., 2007; Feldhaus, 2010). In this camera, each individual pixel in the sensor functions independently and is able to time-stamp an incident 'event', effectively transforming the imaging sensor into an array of fast digitizers with both spatial and temporal resolution. TimepixCam is thus able to detect and time-stamp ion hits with $20 \mathrm{~ns}$ timing resolution, making it possible to record ion time-of-flight (TOF) spectra and ion momentum images for all fragment ions simultaneously, thereby overcoming some of the major limitations of both delay-line detectors and conventional VMI detection techniques. Prior to the experiments reported here, TimepixCam was tested for low-energy ion detection in a variety of experimental conditions with rates of up to at least 200 ion hits per frame (Fisher-Levine \& Nomerotski, 2016). Similar timestamping techniques including the PImMS camera (Nomerotski et al., 2010; Clark et al., 2012; John et al., 2012), which uses monolithic CMOS (complimentary metal-oxide-semiconductor) technology, or approaches based on the direct detection of electrons and ions after a microchannel plate (MCP) employing a Timepix ASIC (Application Specific Integrated Circuit) (Jungmann et al., 2010; Jungmann \& Heeren, 2013; Long et al., 2017), have recently also been used for mass spectrometry applications, including first experiments with free-electron lasers (Amini et al., 2017; Köckert et al., 2018; Brauße et al., 2018). The optical cameras, such as TimepixCam and PImMS, are placed outside of the vacuum, completely decoupled from the whole VMI setup, which brings considerable flexibility for upgrades. TimepixCam also offers a straightforward upgrade path by employing an improved version of the readout chip, Timepix3 (Poikela et al., 2014).

\section{Experimental setup}

The experiment was performed at the CAMP endstation (Strüder et al., 2010; Erk et al., 2018) at beamline BL1 of the soft X-ray free-electron laser facility FLASH (Feldhaus, 2010) at DESY in December 2015. Fig. 1 shows a sketch of the experimental setup. Using a pair of Kirkpatrick-Baez focusing mirrors, the linearly polarized FLASH XUV photon beam (71 or $139 \mathrm{eV}$ photon energy) was focused to a spot size of approximately $10 \mu \mathrm{m} \times 10 \mu \mathrm{m}$ inside the CAMP interaction chamber (Erk et al., 2018), where it crossed a doubly skimmed, continuous supersonic molecular beam of pure $\mathrm{CH}_{2} \mathrm{IBr}$ target molecules that were expanded into the vacuum without using a carrier gas. Linearly polarized femtosecond near-infrared (NIR) and ultraviolet (UV) laser pulses produced as the fundamental $(800 \mathrm{~nm})$ and third harmonic $(267 \mathrm{~nm})$ of the FLASH Ti-Sapphire pump-probe laser (Redlin et al., 2011) were overlapped with the XUV beam in a near-collinear geometry using a drilled dielectric mirror with high-reflectivity coating for broadband 267 and $800 \mathrm{~nm}$ laser pulses. The polarization of all three laser beams was parallel and in the horizontal. The UV and NIR beams were focused by an outof-vacuum lens to spot sizes of approximately $50 \mu \mathrm{m}$ diameter, chosen to be considerably larger than the XUV spot size in order to provide a better stability of the spatial overlap of the UV beam with the FEL beam. The focal lengths for the UV and NIR beams were equalized by adjusting the divergence of the laser beams with lens telescopes. The relative timing between the XUV, NIR and/or UV pulses was controlled by motorized mechanical delay stages such that it was possible to perform NIR-XUV, UV-XUV and UV-NIR pump-probe experiments. Both the FLASH FEL as well as the pumpprobe laser operated at a repetition rate of $10 \mathrm{~Hz}$. From a measurement of the electron bunch duration in the FLASH accelerator (Düsterer et al., 2014; Savelyev et al., 2017), the XUV pulse length was estimated to be $120 \mathrm{fs}$ (FWHM), while

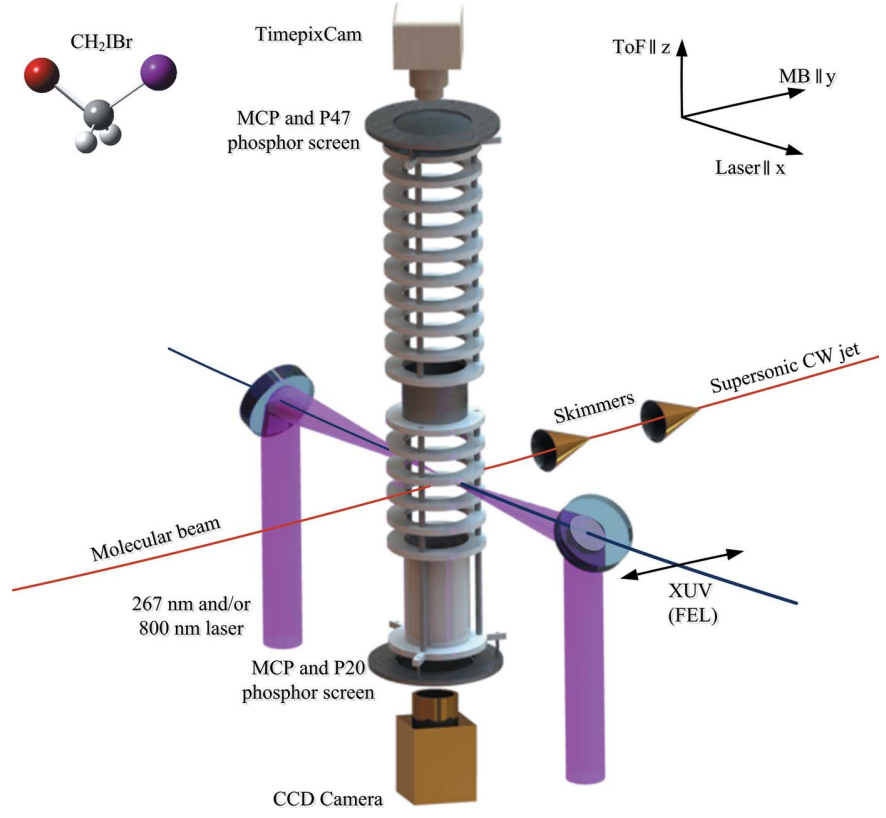

Figure 1

Sketch of the experimental setup showing the molecular beam, the path of the UV, NIR and XUV laser beams, and the double-sided velocity-map imaging spectrometer. Ions were detected with an MCP and a fast P47 phosphor screen detector coupled with TimepixCam. Electrons were detected with an MCP and a P20 phosphor screen detector coupled with a commercial CCD camera. 
the cross correlation of the NIR and UV pulses was measured to be $\sim 170 \mathrm{fs}$ (FWHM) in the interaction region.

The electrons and ions produced by the interaction of the XUV, NIR and/or UV beams with the target molecules were analyzed using a double-sided velocity-map imaging spectrometer (Bomme et al., 2018) equipped with two $80 \mathrm{~mm} \mathrm{MCP}$ phosphor-screen detector assemblies (Photonis APD 2 PS 75/ 32/25/8 I 60:1). The electrons hit the front of the MCP detector with a kinetic energy of approximately $200 \mathrm{eV}$, the ions with a kinetic energy of $2.3 \mathrm{keV}$ per unit charge. For the electrons, a P20 phosphor screen was used, while the ion side of the spectrometer was equipped with a fast P47 phosphor screen. Electron images, which are discussed in a separate publication (Brauße et al., 2018), were recorded at $10 \mathrm{~Hz}$ using a commercial CCD camera (Allied Vision Pike F-145B), while the ion images were recorded with TimepixCam, which is described in detail in the following section. Further details on the data analysis and sorting procedure for pump-probe experiments using the FLASH FEL in combination with the FLASH pump-probe laser, such as the normalization of the data to the fluctuating XUV pulse energy and the correction for the arrival-time jitter between the XUV and the UV/NIR pulses, are given by Savelyev et al. (2017).

To test the performance of TimepixCam, several runs were made under different experimental conditions. The first run ('run 1') was a UV-XUV pump-probe scan at an FEL wavelength of $17.7 \mathrm{~nm}$ (71 eV photon energy) with relatively low FEL and UV power. For this run, the FEL pulses with an average pulse energy of $90 \mu \mathrm{J}$ were attenuated by filters to approximately $2 \mu \mathrm{J}$, and the UV pulse energy on the target was approximately $10 \mu \mathrm{J}$. A second UV-XUV pump-probe scan ('run 2') was taken at $139 \mathrm{eV}$ photon energy and with higher FEL and UV power ( $25 \mu \mathrm{J}$ and $40 \mu \mathrm{J}$, respectively). At these photon energies, the $\mathrm{CH}_{2} \mathrm{IBr}$ molecules are predominantly ionized by the removal of one or several electrons from the $\operatorname{Br}(3 d)$ or $\mathrm{I}(4 d)$ inner shells, followed by Auger decay. Furthermore, a UV-NIR pump-probe scan ('run 3') was taken without FEL, and with the same UV power as in the first run but with a factor of 0.66 lower spectrometer voltages. The NIR pulse energy for this run was $330 \mu \mathrm{J}$.

\subsection{TimepixCam}

The camera is based on a novel silicon sensor with a thin entrance window (Fisher-Levine \& Nomerotski, 2016), read out with a Timepix chip (Llopart et al., 2007), and can efficiently register ions impinging on an MCP in the VMI configuration described above. The MCP is coupled to a fast $\mathrm{P} 47$ phosphor which produces light flashes in response to ions hitting the MCP. The TimepixCam camera, placed outside of the vacuum, can detect light flashes from the P47 with close to $100 \%$ efficiency over the $400-900 \mathrm{~nm}$ wavelength range (Nomerotski et al., 2017). This is a good match to the P47 phosphor emission spectrum, which peaks around $430 \mathrm{~nm}$.

The sensor is bump-bonded to the Timepix ASIC, a timestamping readout chip with $256 \times 256$ pixels, each having a size of $55 \mu \mathrm{m} \times 55 \mu \mathrm{m}$. The processing electronics in each pixel amplify and shape the input charge to record the time of arrival for light flashes ('events') which cross a pre-defined threshold. The minimum achievable threshold is about 700800 photons, depending on the wavelength. In practice, the threshold is tuned in order to achieve negligible noise occupancy in the dark.

Once an event has been detected, it is stored as a timecode in a 14-bit memory inside the corresponding pixel. Each pixel also includes a 4-bit digital-to-analog converter, which allows for equalization of the effective threshold adjustment on a pixel-by-pixel basis. The maximum clock frequency of the chip is $100 \mathrm{MHz}$, corresponding to a $10 \mathrm{~ns}$ clock cycle. However, not all Timepix ASICs operate reliably at this maximum frequency, so TimepixCam is hardwired to use a $20 \mathrm{~ns}$ cycle. Combining this with 11810 cycles (limited by the pseudo 14-bit in-pixel memory) results in a $236 \mu$ s experimental period when the camera 'shutter' is open, and the light flashes from the phosphor can be recorded. In the experiments described here, the duration and timing of the shutter was governed by an external TTL pulse provided to the camera by a pulse generator which was synchronized with the FEL master clock. It should be noted that only one ion hit can be recorded by each pixel during each experimental period.

After each of these experimental periods, i.e. after each laser pulse in a typical imaging mass spectrometry experiment, the data are read out from the chip. The current version of TimepixCam, which employs the UNO readout system for the data acquisition (Imatek, 2016), allows a continuous readout at approximately $10 \mathrm{~Hz}$, limited by the USB connection to the computer and the camera software. The FLASH 'bunch ID', which is an identification number for each FEL pulse, was stored along with the data from each readout cycle and was used to establish the correspondence of the TimepixCam frames and the FLASH bunches so that various intensity and pump-probe timing corrections could be applied (Savelyev et al., 2017). This association was made by the readout software, which recorded the bunch ID in the header of each camera frame.

\section{Characterization of the ion detection and camera performance}

For these measurements, the TimepixCam shutter was configured such that it opened just before the interaction of the laser pulses with the molecular target, in order for the camera to be sensitive to the scattered laser light and subsequently arriving ions. The maximum time of flight of the ions was about $10 \mu$ s. Fig. 2 shows a typical single frame with ion hits, where the color scale denotes the timecodes of the hit pixels in units of $20 \mathrm{~ns}$. Individual ion hits appear as clusters of several pixels with similar timecodes. The ion hits have been identified with a simple cluster-finding algorithm described later. Before running the algorithm, noisy pixels were identified and masked for the rest of the analysis.

Fig. 3 shows the size distribution of the clusters. The most probable size is seven pixels, i.e. the hits are typically two to three pixels across. The peak at four pixels is explained by the 


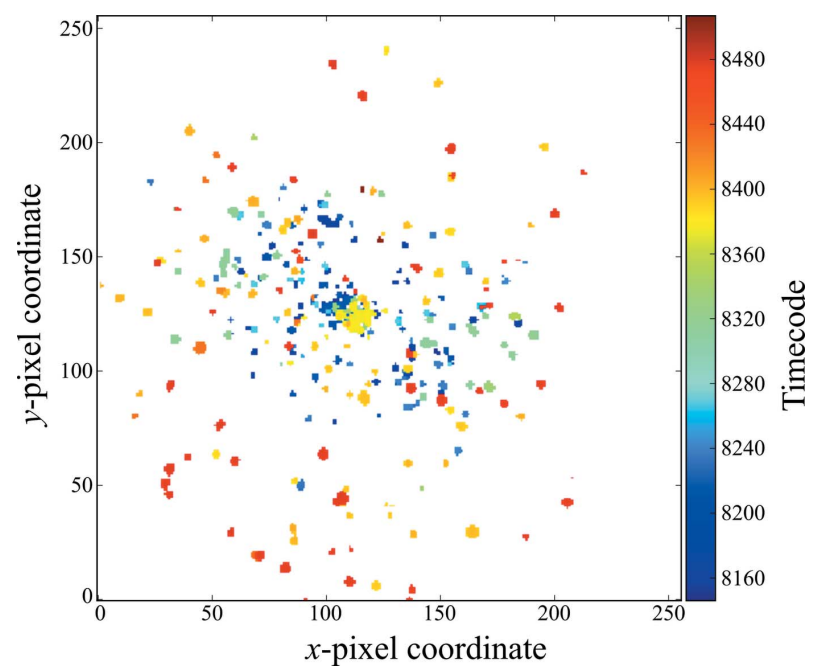

Figure 2

A typical single frame with about 200 ion hits. The color scale denotes the timecodes of the hit pixels in units of $20 \mathrm{~ns}$.

favorable probability of having a $2 \times 2$ pixel cluster due to the symmetric nature of the hits. The large width of the distribution is explained by the width of the MCP electron yield, which has a considerable spread, of the order of $100 \%$ (Hamamatsu, 2007), while the long tail is mostly caused by blended (overlapping) hits. Examples of blended hits can be found later in Fig. 5, and are discussed in more detail below.

The size and shape of the clusters show that there is sufficient signal to allow for centroiding in both time and space, which improves both the spatial and temporal resolutions, as described in more detail below. Note that the cluster size can be controlled by several factors. Assuming that the focusing of the camera lens is optimal, these include the voltage applied across the MCP, the voltage between the MCP and the P47, and the TimepixCam pixel threshold. The settings were chosen to balance the occupancy, cluster finding and centroiding considerations. The detection efficiency of the camera for light

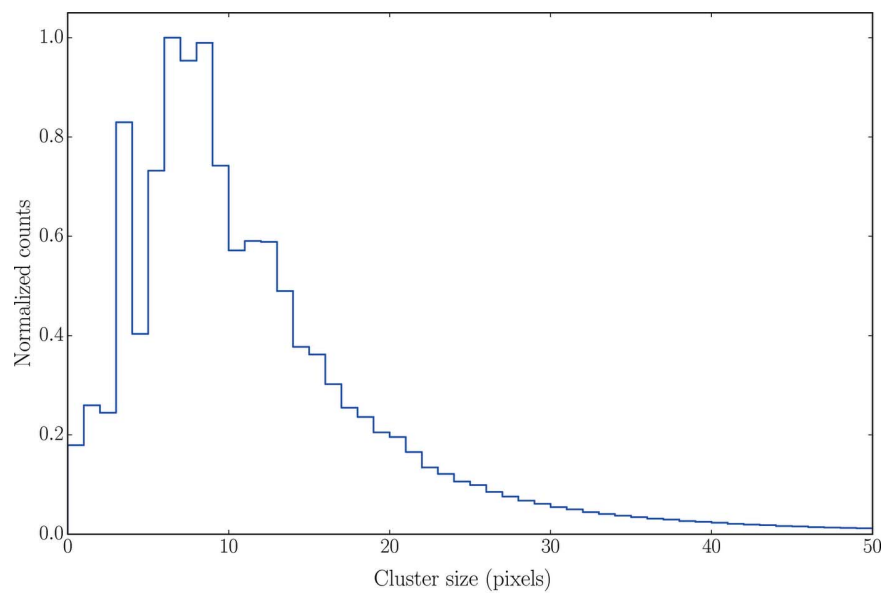

Figure 3

Distribution of the cluster sizes for the reconstructed ion hits. The most probable value is 7 pixels. The peaks at 9 and 4 pixels are due to the geometric favorability of square hits.

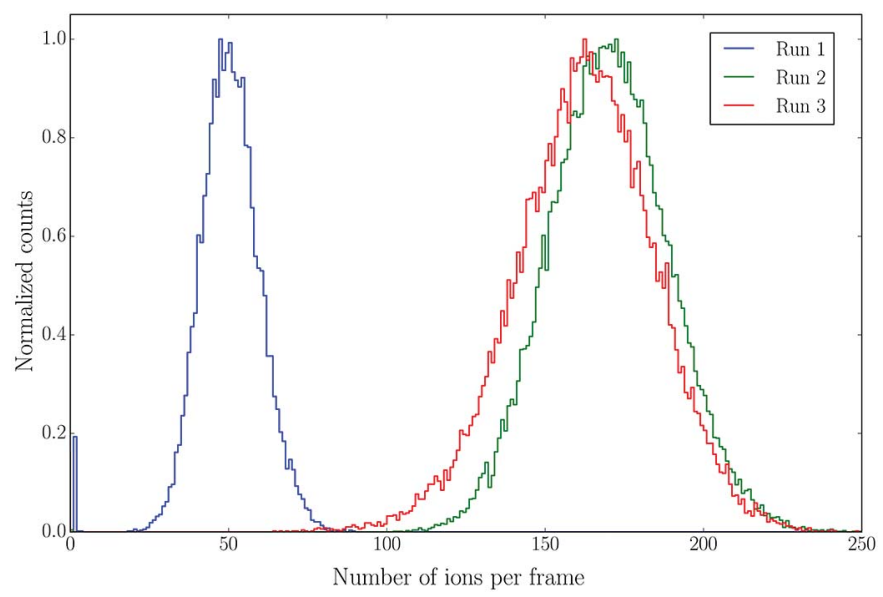

Figure 4

Distribution of the number of reconstructed clusters per frame for the three experimental runs described in the Experimental setup section.

flashes from the phosphor screen is close to $100 \%$ at optimal settings (Nomerotski et al., 2017). Note that this does not, however, take into account the lower MCP detection efficiency for the ions.

The distribution of the number of reconstructed clusters per frame for the three experimental runs described above is shown in Fig. 4. The number of clusters corresponds to the number of ions registered by TimepixCam, with the caveat that some ion hits are blended. Two runs have $\sim 170$ individual ion hits per frame on average, and one run has $\sim 50$ hits per frame. The occupancy of the sensor in the busiest run did not exceed $3 \%$ on average. However, it is highly inhomogeneous, as visible in Fig. 2, which causes some blended hits at the center of the image. These numbers give some indication of how much signal can be comfortably recorded without compromising the quality of the data. At higher occupancies than this, the analysis becomes more complicated due to the large number of blended hits, and is further aggravated by the lack of sensitivity at later times due to the number of pixels being hit early on. The exact maximum count rate that can be tolerated is strongly dependent on the specific experiment, for example how frequent a given ionic species is detected, and on how these ions are spatially distributed on the detector.

Ion hits are identified as contiguous areas of pixels with non-zero timecodes where the signal was high enough to cross the threshold and measure the time of arrival. They are typically round clusters with a diameter of a few pixels. As can be seen in Fig. 5, each ion has a distribution of time codes with the fastest response in the center. This is caused by a larger light flux in the hit center, and hence a faster rise time in the middle of the cluster and correspondingly slower rise time in the surrounding pixels. As each cluster corresponds to one ion, which only has one arrival time, a centroiding algorithm was employed that assigns the earliest arrival time in the cluster to the center of mass of the event. The center of mass is calculated using all pixels in the cluster. This significantly improves both the spatial and the timing resolution of the detector, as shown in Figs. 5 and 6. 



Figure 5

Examples of several ion hits. The color-scale marks the timecode of the hit pixels. The top two plots show isolated hits, the second two show blended hits that are separate in time, i.e. belonging to different ion species, while the bottom two show the blending of hits of the same ion species.

blend together, as demonstrated in the examples shown in Figs. 5(c)-5(f). If the two ions have different time of flight, as is apparent in Figs. 5(c) and 5(d), their separation is straightforward. Note that Timepix currently does not have multihit capability in a given pixel, i.e. each pixel can measure time only once per frame, so if a pixel is hit once it cannot record a second hit in the same frame. If two ions have a similar mass-to-charge ratio, the hit timecodes are similar, as shown in Figs. 5(e) and $5(f)$. The examples were chosen to illustrate a variety of cases in which blended clusters are formed. The separation of the blends requires a complex clustering algorithm, the performance of which will depend on the cluster size, overall chip occupancy, and the particulars of the TOF spectrum. While the development of such a deblending code is in progress, the results shown here were obtained using a simplified version, which does not attempt to separate the blended hits.

The ion TOF spectrum for one of the runs using the XUV FEL and the UV laser pulse ('run 1') is shown in Fig. 7. The TOF spectrum obtained from TimepixCam after application of the centroiding algorithm is shown along with the spectrum obtained directly from the decoupled analog MCP signal, recorded in parallel with a high-resolution digitizer (SP Devices ADQ412AC4 G-MTCA) with 0.5 ns timing resolution. Although the spectrum obtained from the MCP signal has a slightly better mass resolution, all major peaks can be separated in the TimepixCam spectrum, demonstrating that the temporal resolution of TimepixCam is

Fig. 5 shows several examples of ion hits in an attempt to classify various cluster types since the appearance of ions varies considerably due to the random nature of the size variation, blending, ion species' spatial distribution and the presence of multiple ions species in the frames. Single ion hits can vary in size, but typically have symmetric shapes as shown in Figs. 5(a) and 5(b) since the generation of electrons in the MCP and the emission of photons in the phosphor are symmetric. This can be used to improve the position resolution by applying a spatial centroiding algorithm. The timecodes for the hit pixels also have a profile with a maximum in the center corresponding to the hits with the maximum signal. They correspond to the earliest hits and should be used as an estimate of the ion time of flight. However, two ion hits can also sufficient for typical ion VMI applications. We further note that the TimepixCam spectrum is not affected by ringing and other high-frequency noise present in the MCP signal due to imperfect impedance matching in the signal outcoupling. A double-peak structure due to stray light from the FEL and the UV-laser is present early in the TimepixCam spectrum. It is also present in the MCP signal but was cut in this figure since it created a large amount of ringing.

The time resolution of TimepixCam for the given experimental conditions was estimated by analyzing the $\mathrm{H}^{+}$ion mass peak using the time centroiding algorithm described above and fitting the mass peak with a Gaussian distribution. Fig. 6 shows the timing resolution for the raw hits and the resolution after the centroiding algorithm. The Gaussian fits of the $\mathrm{H}^{+}$ 

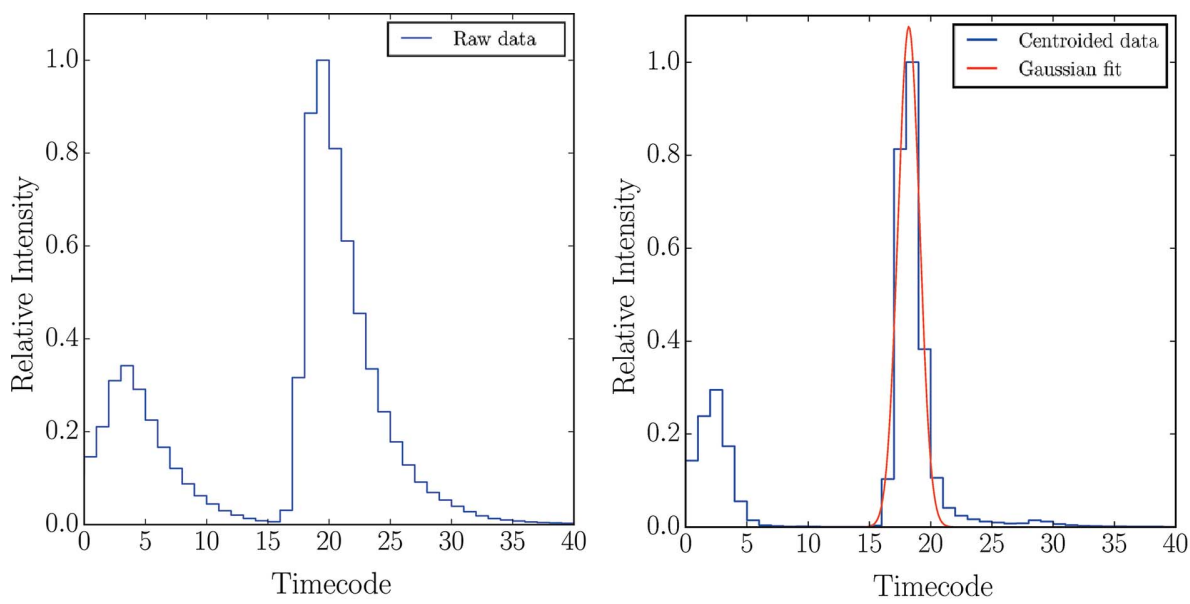

Figure 6

Timing resolution determined for the raw $\mathrm{H}^{+}$ion hits (left), and the resolution after a time centroiding algorithm has been applied (right). The $\mathrm{H}^{+}$peak is fit with a Gaussian distribution, as described in the text. The smaller peaks preceding the $\mathrm{H}^{+}$ion peak by about $300 \mathrm{~ns}$ arise from the scattered laser photons.

ion peaks have a $\sigma$ value of $34.8 \mathrm{~ns}$ and $17.7 \mathrm{~ns}$ before and after centroiding, respectively, demonstrating the improvement in timing resolution due to the centroiding. In the former case, the Gaussian function is not a good representation of the peak shape and is not shown. We note that the achieved timing resolution for $\mathrm{H}^{+}$is likely to be limited by the camera, mostly due to the so-called chess-board effect, which can vary the timecode by one count in either direction (Nomerotski et al., 2017). We also note that some of the mass peaks have additional broadening due to the momentum of the ions, as can be seen in the high-resolution spectrum obtained from the decoupled analog MCP signal shown in Fig. 7.

\section{Probing the UV-induced dissociation dynamics of $\mathrm{CH}_{2} \mathrm{IBr}$}

To demonstrate the capabilities and advantages of combining TimepixCam with a velocity-map imaging setup for pumpprobe experiments with free-electron lasers and optical lasers, we present data on the UV-induced dissociation of gas-phase $\mathrm{CH}_{2} \mathrm{IBr}$ probed by time-resolved Coulomb explosion imaging as an example. A more detailed discussion of the results and conclusions from the experiment is given by Burt et al. (2017) and Köckert et al. (2018).

For the data shown here, the $\mathrm{CH}_{2} \mathrm{IBr}$ molecules were photodissociated by absorption of a single UV photon at $267 \mathrm{~nm}$, and the dissociation dynamics were then probed after an adjustable delay, $\tau$, by an intense light pulse at either NIR or XUV wavelengths. The intense $800 \mathrm{~nm}$ NIR pulse strong-field ionizes the molecule, which then breaks into several singly or multiply charged cations that are emitted with kinetic energies of several electronvolts per fragment due to the Coulomb repulsion between the charged fragments. Absorption of one or several XUV photons also creates multiple charged fragments with high kinetic energies up to a few tens of electronvolts. At the photon energies of 71 and $139 \mathrm{eV}$ chosen here, the $\mathrm{CH}_{2} \mathrm{IBr}$ molecules are predominantly ionized by the removal of one or several electrons from the $\operatorname{Br}(3 d)$ or $\mathrm{I}(4 d)$ inner shells, followed by Auger decay.

The resulting ion TOF spectrum and the corresponding fragment ion images recorded with TimepixCam are shown in Figs 7, 8 and 9. In Fig. 8, the ion images for each fragment are shown next to the TOF spectrum obtained after ionization with the NIR pulse. By recording the images for all ion species simultaneously, which is possible using a fast time-stamping camera such as TimepixCam, the momentum distributions for all fragments can be compared. While the distributions of the $\mathrm{H}^{+}$ions and of the carbon containing fragments are mostly isotropic, the momentum distributions of the highly charged bromine and iodine ions exhibit a clear anisotropy with maxima along the polarization direction of the laser pulses since the ionization and fragmentation probability is highest for molecules whose $\mathrm{I}-\mathrm{C}$ bond axis is parallel to the polarization direction of the NIR field. The bromine and iodine ion images display features at different radii, corresponding to different kinetic energy channels, as can be seen in more detail in Fig. 9. In the ion images from the UV-NIR pump-probe run (top row in Fig. 9), the two outer lobes correspond to the Coulomb explosion of intact $\mathrm{CH}_{2} \mathrm{IBr}$ molecules into a $\mathrm{Br}^{+}$or $\mathrm{I}^{2+}$ ion and a singly or doubly charged co-fragment. The feature at smaller radii corresponds to a lower kinetic energy of the $\mathrm{Br}^{+}$or $\mathrm{I}^{2+}$ ions, stemming from $\mathrm{Br}^{+}$

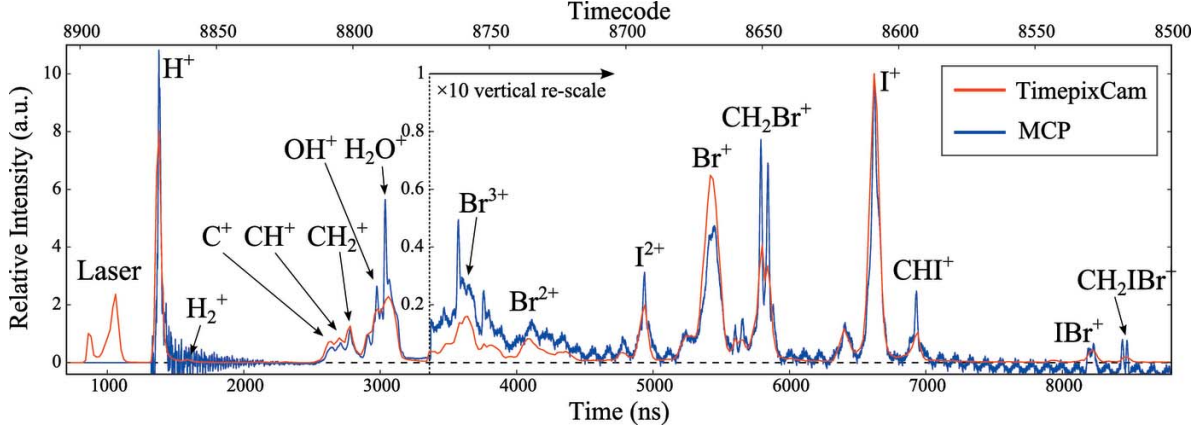

Figure 7

Ion TOF mass spectra for the ionization of $\mathrm{CH}_{2} \mathrm{IBr}$ with an intense $71 \mathrm{eV}$ XUV pulse, recorded with TimepixCam (red line), and overlaid with the spectrum obtained directly from the decoupled analog MCP signal, recorded with a high-resolution digitizer (blue line). Both UV and XUV pulses were present for this run, and the spectra are integrated over all pump-probe delays. The spectra are normalized to the maximum of the $\mathrm{I}^{+}$peak. TimepixCam timecodes are shown on the top horizontal axis, with the lower horizontal axis showing the time in nanoseconds. 


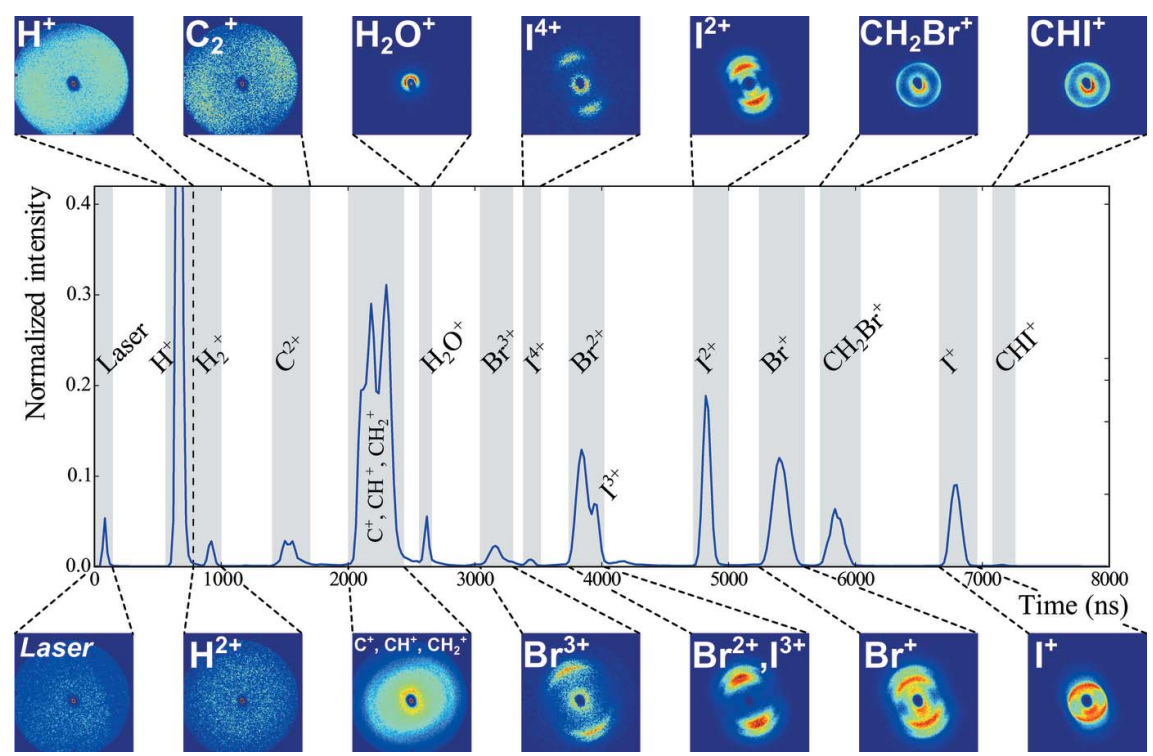

Figure 8

Ion TOF mass spectrum of $\mathrm{CH}_{2} \mathrm{IBr}$ recorded with TimepixCam after strong-field ionization with an intense $800 \mathrm{~nm}$ pulse (blue line) along with the camera images for each of the peaks in the TOF spectrum. Both UV and NIR pulses were present for this run, and the spectrum is integrated over all pump-probe delays. The (linear) polarization direction of the UV and NIR laser pulses was parallel to the ion detector and at an angle of approximately $-30^{\circ}$ with respect to the vertical axis in these images, which are integrated over a total of 35000 laser shots. The dark spot at the center of the detector and the intense pixel in the center for ions of mass 18 and lighter correspond to an area where overlapping hits were not properly identified by the centroiding algorithm due to the intense spatially localized water peak.
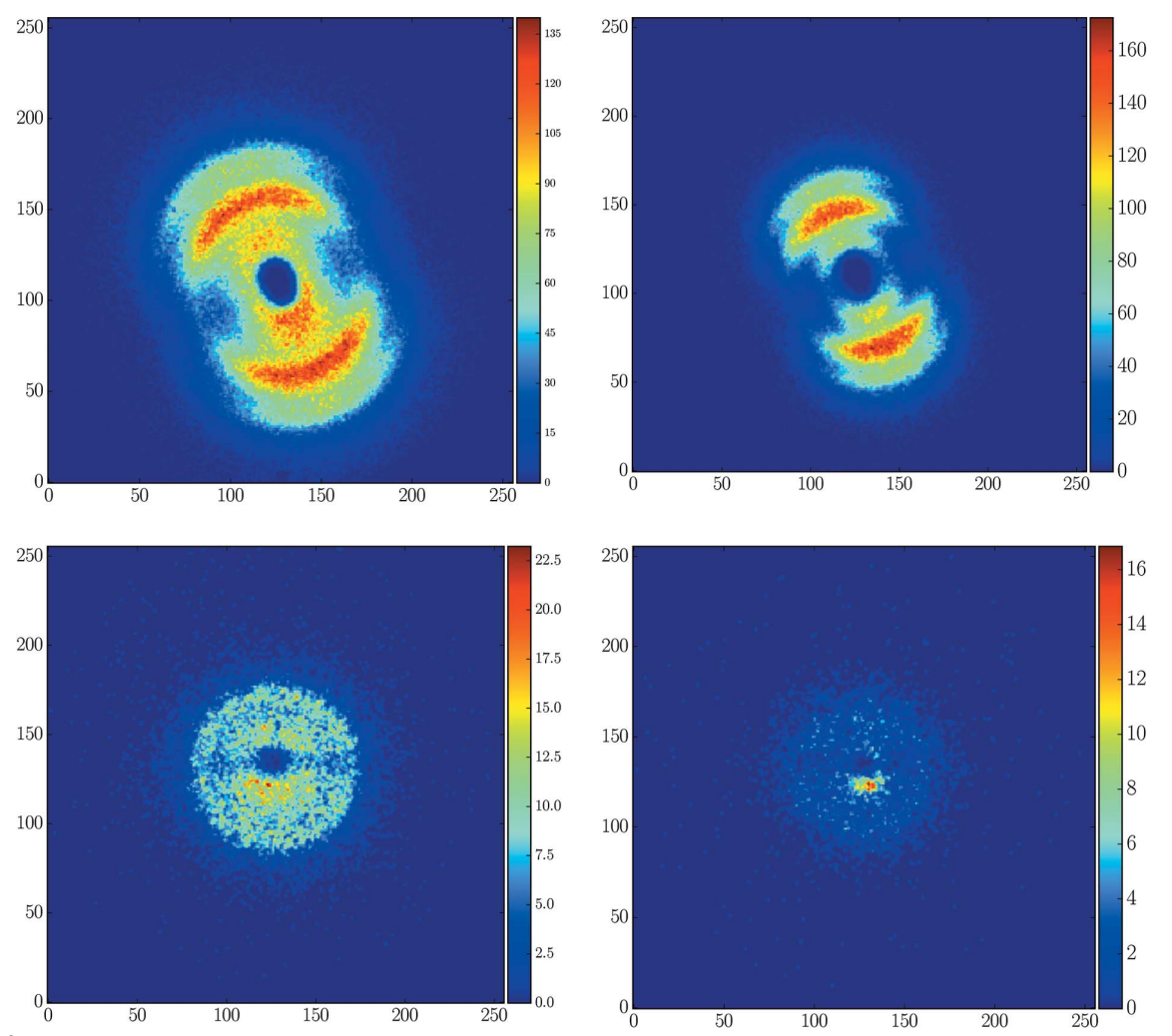

Figure 9

Ion images of $\mathrm{Br}^{+}$(left) and $\mathrm{I}^{2+}$ (right) fragments created after ionization with 35000 NIR laser pulses (top) and $12000 \mathrm{XUV}$ pulses at $71 \mathrm{eV}$ photon energy (bottom). The images are integrated over all pump-probe delays. The (linear) polarization direction of the UV, NIR and XUV pulses was parallel to the ion detector and at an angle of approximately $-30^{\circ}$ with respect to the vertical axis in all of these images. or $\mathrm{I}^{2+}$ ions that are produced with a neutral co-fragment, for example if the molecule is first UV-dissociated and then post-ionized by the NIR pulse (Burt et al., 2017). This feature is also visible in the images from the UV-FEL pump-probe run (bottom row in Fig. 9), which were taken at higher spectrometer voltages and which are thus more compressed. The ion distributions produced by the FEL are more isotropic than those produced by the NIR laser since the inner-shell ionization probability is much less dependent on the orientation of the molecular axes. The pronounced 'hole' in the center of the images and the weaker horizontal stripe that is visible in the images in the bottom row of Fig. 9 is due to saturation of the corresponding pixels by ions that arrived at earlier times within the same acquisition cycle.

In order to show the quality of the raw data directly as it is recorded, the images in Figs. 8 and 9 are shown without any inversion procedure, i.e. they correspond to the two-dimensional projections of the three-dimensional momentum distributions on the detector plane, which is parallel to the light propagation direction as well as to the polarization vectors of the UV, NIR and XUV pulses. We note that the time-stamping of each ion hit allows the slice imaging technique to be applied (Amini et al., 2015), thereby making it possible to obtain two-dimensional slices through the three-dimensional ion momentum distribution even for experimental geometries that do not have cylindrical symmetry. Such timesliced images of the $\mathrm{I}^{2+}$ ion distribution are shown in Fig. 10. The panel in the middle shows the central slice through the distribution, while the earlier and later images correspond to $\mathrm{I}^{2+}$ ions emitted towards or away from the ion detector. The up-down asymmetry in some of these images is an indication that the polarization vector in this experimental run was not perfectly parallel to the detector surface.

To investigate the dynamics of the dissociation process, Fig. 11 shows the radial projection of the integrated (i.e. not sliced) $\mathrm{I}^{2+}$ ion images as a function of the delay between the UV-pump and 

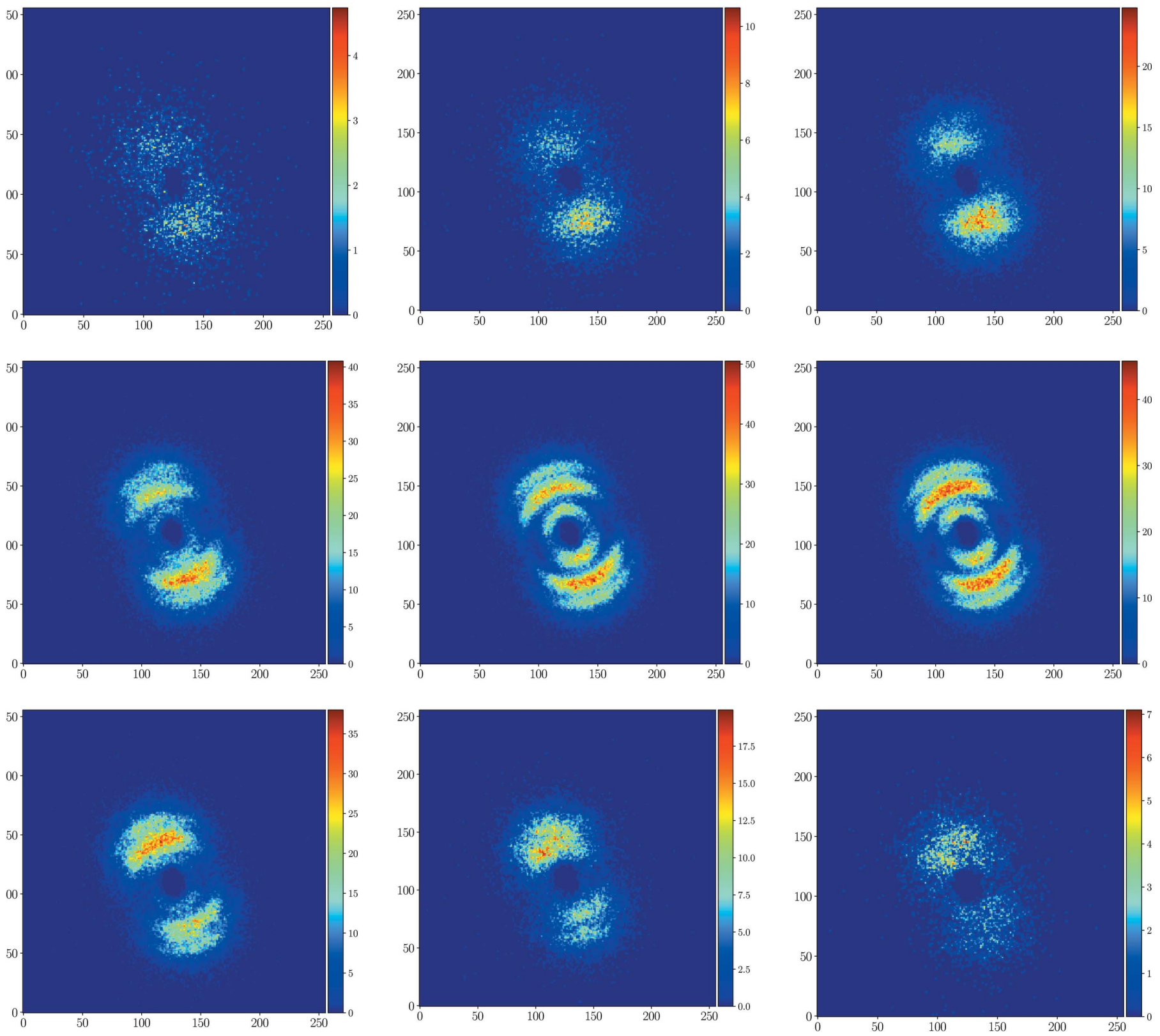

Figure 10

Time sliced $\mathrm{I}^{2+}$ ion images showing the same data for $\mathrm{I}^{2+}$ ions as in the previous figure but for each time code within the $\mathrm{I}^{2+}$ peak separately. From top left to bottom right, the time codes are 8264 through 8272 , with each time code representing a $20 \mathrm{~ns}$ time bin.

the FEL-probe pulses. If the UV pulse precedes the FEL pulse, a contribution close to the center of the image (i.e. at small radii) appears, corresponding to ions that are created with low kinetic energies, as discussed above. As explained in detail in other studies (Erk et al., 2014; Boll et al., 2016; Savelyev et al., 2017), these low-kinetic ions are produced when the $\mathrm{CH}_{2} \mathrm{IBr}$ molecule is first dissociated, via cleavage of the $\mathrm{C}-\mathrm{I}$ bond, into two neutral fragments by the UV pulse, and the iodine fragment is subsequently ionized by the FEL pulse. Similar delay-dependent spectra were recorded simultaneously for all ionic fragments. A more detailed analysis of the time-dependent effects and the UV-induced dissociation dynamics will be presented by Köckert et al. (2018), since the present paper is focused on the technical aspects of using TimepixCam for free-electron laser experiments.

\section{Conclusions}

The first application of TimepixCam for velocity-map ion imaging at a free-electron laser has been reported. TimepixCam is able to simultaneously record the TOF spectrum and the two-dimensional momentum distributions of all ionic fragments and can cope with count rates of several hundred ions per shot. As most experiments at the currently operating FEL facilities are performed at a low repetition rate but high pulse energy, meaning that a large number of ions are 


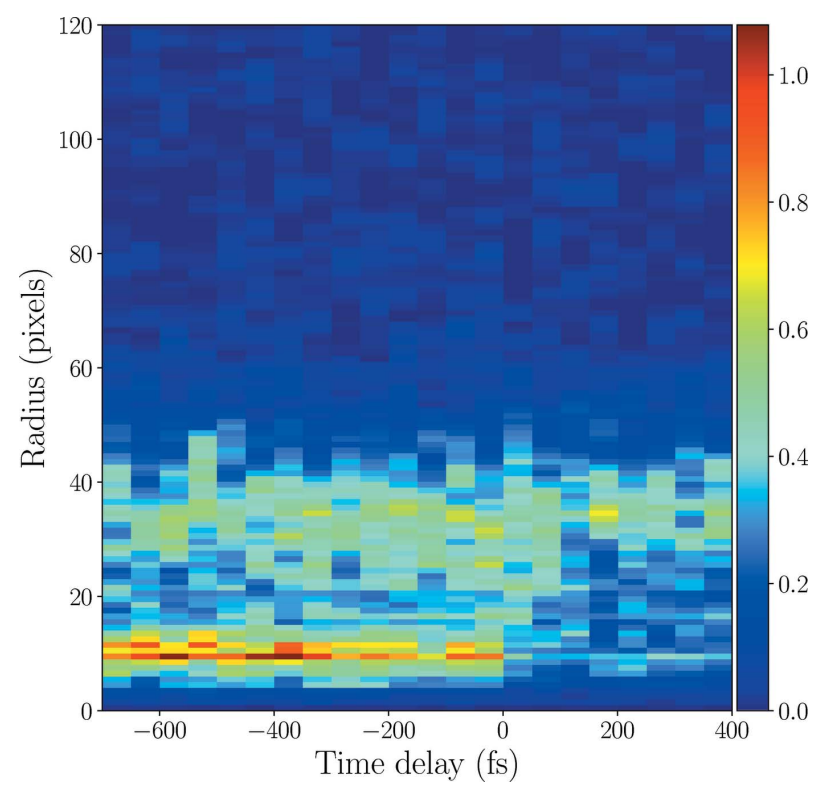

Figure 11

Radial projection of the $\mathrm{I}^{2+}$ ion yield as a function of pump-probe delay between the UV and the $71 \mathrm{eV}$ FEL pulses. Negative delays refer to the UV pulse arriving before the FEL pulse. The yield for each delay step is normalized on the shot-by-shot pulse energy of the FEL.

produced within a single FEL shot, the combination of fast time-stamping cameras such as TimepixCam or PImMS (John et al., 2012; Amini et al., 2017; Köckert et al., 2018) with a VMI spectrometer offer experimental capabilities that are difficult to achieve otherwise. The ability to detect ion images for all fragments simultaneously is of particular advantage for timedependent pump-probe studies, where drifts, for example in the timing and pulse energy, severely limit the comparability of pump-probe scans for different fragments that are recorded consecutively. Moreover, it also allows ion-ion covariance or coincidence techniques to be applied to determine momentum correlations between fragments (Slater et al., 2014; Zhao et al., 2017). Combining TimepixCam with velocity-map ion imaging thus enhances significantly the applicability of XUV-induced Coulomb explosion imaging experiments to investigate ultrafast molecular dynamics in gas-phase molecules.

A major disadvantage of the present version of TimepixCam is the limitation to one detected ion per pixel per detection cycle, which can lead to severe saturation effects, especially for heavier ions that arrive at long flight times and thus late in the detection cycle. In order to overcome this limitation and to increase the timing resolution of the camera, TimepixCam is being upgraded to use the Timepix3 ASIC, a new generation of time-stamping chip (Poikela et al., 2014). The thin window sensors of TimepixCam are fully compatible with Timepix3, which has the same pixel geometry as the previous version of the chip, while the timing resolution is improved by an order of magnitude to $1.5 \mathrm{~ns}$. Each pixel is able to time-stamp hits with only $0.5 \mu$ s dead-time, thereby providing multi-hit capabilities, which is another major improvement over the previous Timepix chip. The small deadtime allows genuine three-dimensional separation of ions in the $(x, y$, time) space removing limitations caused by the pile- up in the $(x, y)$ projection. Furthermore, the Timepix 3 readout is data driven and supports high rates up to $80 \mathrm{Mpixel} \mathrm{s}^{-1}$ with existing readout systems such as SPIDR (Visser et al., 2015). This rate corresponds to $\sim 8$ million ions per second, or to 40 thousand frames (i.e. laser shots) per second, assuming 10 hit pixels per ion and 200 ions per frame. First experiments using the Timepix3-based TimepixCam are in progress (Zhao et al., 2017).

Besides promising much higher-quality data for experiments such as the one presented in this manuscript, the nextgeneration TimepixCam also opens up a new application in the context of ion and electron imaging experiments at highrepetition-rate free-electron lasers such as the European XFEL, which will deliver up to 2700 pulses per pulse train with only $220 \mathrm{~ns}$ spacing (Tschentscher et al., 2017). This burst mode operation poses a significant challenge to the data acquisition and readout. Even for dilute gas-phase molecular targets, electron or ion hit rates in Coulomb-explosion imaging experiments can easily reach several hundreds of particles per shot, as a single X-ray FEL pulse can create multiple charged particles from a single small molecule. Delay-line anodes are not able to cope with this many hits in a short time, and CCD cameras, typically read out at 10 to a few hundred $\mathrm{Hz}$, are only able to record an integrated image for all pulses of a train. Under these conditions, time-stamping cameras such as TimepixCam or PImMS can be of great advantage for both, ion and electron VMI experiments since it is often necessary to conduct data analysis on a single-shot basis (Savelyev et al., 2017). The time-stamping can be exploited to record individual single-shot electron detector images for each pulse of the train (i.e. spaced by $220 \mathrm{~ns}$ ). For ions, flight times depend strongly on the molecular mass and the applied spectrometer voltages, but can often be up to $10 \mu \mathrm{s}$, such that the pulse spacing needs to be increased if overlapping mass spectra shall be avoided. Nevertheless, a time-stamping camera can either be used to record all fragments simultaneously, as described herein, or, if hit rates are too high and/or the resolution for small masses is poor due to deliberately short flight times, it can be combined with fast switching/gating of the ion detector, such that only one or few specific ion masses are detected for each photon pulse. Both applications are considered for the upcoming Small Quantum Systems (SQS) instrument at the European XFEL (Tschentscher et al., 2017).

\section{Acknowledgements}

We gratefully acknowledge the work of the scientific and technical team at FLASH, who made these experiments possible. We also acknowledge the Max Planck Society for funding the development of the CAMP instrument within the ASG at CFEL. We thank Erland Müller, Evgeny Savelyev, Sadia Bari, Bastian Manschwetus, Joss Wiese, Jochen Küpper Artem Rudenko, Felix Brausse, Gildas Goldsztejn, Arnaud Rouzée, Per Johnson, Sylvain Maclot, Jan Lahl, Pavel K. Olshin, Andrey S. Mereshchenko, Jonathan Underwood, David Holland, Thierry Ruchon, Romain Geneaux, Marie Geleoc, Jérôme Palaudoux, Francis Penent, Pascal Lablan- 
quie, Barbara Cunha de Miranda, Iyas Ismail, Maria N. Piancastelli, Oksana Travnikova, Loïc Journel, Renaud Guillemin, Marc Simon, Hansjochen Köckert, Michael Burt, Kasra Amini, Jason W. L. Lee and Alexander Gentleman for their help during the beam time.

\section{Funding information}

Funding for this research was provided by: Chemical Sciences, Geosciences, and Biosciences Division, Office of Basic Energy Sciences, Office of Science, US Department of Energy (award No. DE-FG02-86ER13491 to DRol, FZ); HelmholtzGemeinschaft through the Helmholtz Young Investigator Program (award to DRol, RB, CB, BE); Brookhaven National Laboratory (LDRD grant No. 13-006 to MFL, AN).

\section{References}

Ablikim, U., Bomme, C., Xiong, H., Savelyev, E., Obaid, R., Kaderiya, B., Augustin, S., Schnorr, K., Dumitriu, I., Osipov, T., Bilodeau, R., Kilcoyne, D., Kumarappan, V., Rudenko, A., Berrah, N. \& Rolles, D. (2016). Sci. Rep. 6, 38202.

Ackermann, W. et al. (2007). Nat. Photon. 1, 336-342.

Allaria, E. et al. (2012). Nat. Photon. 6, 699-704.

Allaria, E. et al. (2013). Nat. Commun. 4, 2476.

Amini, K., Blake, S., Brouard, M., Burt, M., Halford, E., Lauer, A., Slater, C., Lee, J. \& Vallance, C. (2015). Rev. Sci. Instrum. 86, 103113.

Amini, K. et al. (2017). J. Chem. Phys. 147, 013933.

Boll, R. et al. (2016). Struct. Dyn. 3, 043207.

Bomme, C. et al. (2018). In preparation.

Brauße, F. et al. (2018). Submitted.

Burt, M. et al. (2017). Phys. Rev. A, 96, 043415.

Chini, M., Zhao, K. \& Chang, Z. (2014). Nat. Photon. 8, 178-186.

Clark, A. T. et al. (2012). J. Phys. Chem. A, 116, 10897-10903.

Dörner, R., Mergel, V., Jagutzki, O., Spielberger, L., Ullrich, J., Moshammer, R. \& Schmidt-Böcking, H. (2000). Phys. Rep. 330, 95192.

Düsterer, S. et al. (2014). Phys. Rev. ST Accel. Beams, 17, 120702.

Emma, P. et al. (2010). Nat. Photon. 4, 641-647.

Eppink, A. T. J. B. \& Parker, D. H. (1997). Rev. Sci. Instrum. 68, $3477-$ 3484.

Erk, B. et al. (2014). Science, 345, 288-291.

Erk, B. et al. (2018). In preparation.

Feldhaus, J. (2010). J. Phys. B, 43, 194002.

Fisher-Levine, M. \& Nomerotski, A. (2016). J. Instrum. 11, C03016.

Hädrich, S., Klenke, A., Rothhardt, J., Krebs, M., Hoffmann, A.,

Pronin, O., Pervak, V., Limpert, J. \& Tünnermann, A. (2014). Nat. Photon. 8, 779-783.

Hamamatsu (2007). Photomultiplier Tubes, Basics and Applications, 3rd ed., ch. 10, p. 200. Hamamatsu Photonics KK, Japan.
Imatek (2016). X-ray Imatek S. L., Eureka Building, UAB Campus, ES-08193 Bellaterra, Barcelona, Spain.

Ishikawa, T. et al. (2012). Nat. Photon. 6, 540-544.

John, J. J. et al. (2012). J. Instrum. 7, C08001.

Jungmann, J. H. \& Heeren, R. M. A. (2013). Rapid Commun. Mass Spectrom. 27, 1-23.

Jungmann, J. H., MacAleese, L., Buijs, R., Giskes, F., de Snaijer, A., Visser, J., Visschers, J., Vrakking, M. J. J. \& Heeren, R. M. A. (2010). J. Am. Soc. Mass Spectrom. 21, 2023-2030.

Köckert, H. et al. (2018). In preparation.

Llopart, X., Ballabriga, R., Campbell, M., Tlustos, L. \& Wong, W. (2007). Nucl. Instrum. Methods Phys. Res. A, 581, 485-494.

Long, J., Furch, F. J., Durá, J., Tremsin, A. S., Vallerga, J., Schulz, C. P., Rouzée, A. \& Vrakking, M. J. J. (2017). J. Chem. Phys. 147, 013919.

Nomerotski, A., Brouard, M., Campbell, E., Clark, A., Crooks, J., Fopma, J., John, J. J., Johnsen, A. J., Slater, C., Turchetta, R., Vallance, C., Wilman, E. \& Yuen, W. H. (2010). J. Instrum. 5, C07007.

Nomerotski, A., Chakaberia, I., Fisher-Levine, M., Janoska, Z., Takacs, P. \& Tsang, T. (2017). J. Instrum. 12, C01017.

Pešić, Z., Rolles, D., Perri, M., Bilodeau, R., Ackerman, G., Rude, B., Kilcoyne, A., Bozek, J. \& Berrah, N. (2007). J. Electron Spectrosc. Relat. Phenom. 155, 155-159.

Poikela, T. et al. (2014). J. Instrum. 9, C05013.

Popmintchev, T., Chen, M.-C., Arpin, P., Murnane, M. M. \& Kapteyn, H. C. (2010). Nat. Photon. 4, 822-832.

Redlin, H., Al-Shemmary, A., Azima, A., Stojanovic, N., Tavella, F., Will, I. \& Düsterer, S. (2011). Nucl. Instrum. Methods Phys. Res. A, 635, S88-S93.

Rolles, D., Pesic, Z., Perri, M., Bilodeau, R., Ackerman, G., Rude, B., Kilcoyne, A., Bozek, J. \& Berrah, N. (2007). Nucl. Instrum. Methods Phys. Res. B, 261, 170-174.

Savelyev, E. et al. (2017). New J. Phys. 19, 043009.

Shintake, T. et al. (2008). Nat. Photon. 2, 555-559.

Slater, C. S., Blake, S., Brouard, M., Lauer, A., Vallance, C., John, J. J., Turchetta, R., Nomerotski, A., Christensen, L., Nielsen, J. H., Johansson, M. P. \& Stapelfeldt, H. (2014). Phys. Rev. A, 89, 011401.

Strüder, L. et al. (2010). Nucl. Instrum. Methods Phys. Res. A, 614, 483-496.

Tschentscher, T., Bressler, C., Grünert, J., Madsen, A., Mancuso, A. P., Meyer, M., Scherz, A., Sinn, H. \& Zastrau, U. (2017). Appl. Sci. 7, 593.

Ullrich, J., Moshammer, R., Dorn, A., Dörner, R., Schmidt, L. P. H. \& Schmidt-Böcking, H. (2003). Rep. Prog. Phys. 66, 1463-1545.

Ullrich, J., Rudenko, A. \& Moshammer, R. (2012). Annu. Rev. Phys. Chem. 63, 635-660.

Visser, J., van Beuzekom, M., Boterenbrood, H., van der Heijden, B., Muñoz, J. I., Kulis, S., Munneke, B. \& Schreuder, F. (2015). J. Instrum. 10, C12028.

Zhao, A., van Beuzekom, M., Bouwens, B., Byelov, D., Chakaberia, I., Cheng, C., Maddox, E., Nomerotski, A., Svihra, P., Visser, J., Vrba, V. \& Weinacht, T. (2017). Rev. Sci. Instrum. 88, 113104. 